\title{
Spectrum sensing of wideband signals based on cyclostationary detection and compressive sensing
}

\author{
Ali Mohammad A. Al-Hussain, Maher K. Mahmood Al Azawi \\ Electrical Engineering Department, Collage of Engineering, Al Mustansiriyah University, Iraq
}

\begin{abstract}
Article Info
Article history:

Received Nov 4, 2019

Revised Mar 13, 2020

Accepted May 1, 2020

\section{Keywords:}

Compressive sensing

Cyclostationary detection

Spectrum sensing

ABSTRACT

Compressive sensing is a powerful technique used to overcome the problem of high sampling rate when dealing with wideband signal spectrum sensing which leads to high speed analogue to digital convertor (ADC) accompanied with large hardware complexity, high processing time, long duration of signal spectrum acquisition and high consumption power. Cyclostationary based detection with compressive technique will be studied and discussed in this paper. To perform the compressive sensing technique, discrete cosine transform (DCT) is used as sparse representation basis of received signal and Gaussian random matrix as a sensing matrix, and then $\ell 1$ - norm recovery algorithm is used to recover the original signal. This signal is used with cyclostationary detector. The probability of detection as a function of SNR and the probability of false alarm as a function of SNR with several compression ratios and processing time are used as performance parameters. The effect of the recovery error of reconstruction algorithm is presented as a function of probability of detection. Simulation results show that the performance of the system is maintained even at high compression.
\end{abstract}

Copyright $@ 2020$ Institute of Advanced Engineering and Science. All rights reserved.

\section{Corresponding Author:}

Ali Mohammad A.AL-Hussain, Electrical Engineering Department, Al Mustansiriyah University, Baghdad, Iraq. Email:alinussaif@yahoo.com

\section{INTRODUCTION}

Scarcity of available radio spectrum due to inefficient utilization based on fixed spectrum allocation policy (FSA) produces problems to be solved associated with the fast growth of communication applications. Several solutions are suggested to overcome this issue, one them is cognitive radio (CR).Spectrum sensing technique is the essential function of cognitive radio and more challenges are present associated with this technique. The most significant of them is wideband signal spectrum sensing with fast time of acquisition in specific region to meet optimal opportunities exploitation of available radio spectrum. Cognitive radio system (CR) involves digital signal processing operation called spectrum sensing technique in order to detect the radio spectrum opportunities to allow the secondary user to access a white space of licensed user frequency band [1,2]. CR is an intelligent wireless communication system that can adapt its parameters depending on the surrounding electromagnetic environment conditions to improve the efficiency of available radio spectrum [3]. It should have ability to sense, measure and recognize the characteristics of the interested channel [4]. This function is implemented by reconfigurable wireless communication system called Software Defined Radio (SDR) [5, 6].

Spectrum sensing is achieved by several methods, such as energy detection (ED) [7, 8], and cyclostationary detection (CSD) [9, 10] matched filter detection (MFD) [11, 12]. The main task in cognitive radio is spectrum sensing. This is required to detect white space of the available spectrum. This function is associated with many challenges and problems. The main challenge here is the sense of a wideband signal since high sampling rate is required and hence high speed ADC is required to perform this function. This 
process is difficult in implementation, has high processing time and required high power. To overcome this problem, compressive sensing techniques (CS) is a promising technique. The concept of compressive sensing is firstly viewed by Candes, Tao [13], Romberg and Donoho [14]. It states that any sparse signal with any sparse basis or compressible signal can be recovered by using non-adaptive measurement of small number elements vector called Measurement vector [15]. Compressive sensing consists of three steps, these are: acquisition of compressed measurements, recovery of the original signal with Nyquist rate sampling and finally, choosing spectrum sensing technique using the reconstructed signal from the previous steps. Since compressive sensing performs spectrum sensing of wideband signal by using smaller number of measurements say $(\mathrm{M})$ from the wideband signal instead of whole signal say $(\mathrm{N})$ elements, where $\mathrm{N} \gg \mathrm{M}$, by using suitable signal recovery algorithm [16]. $\ell 1$ - norm algorithm is used to recover the original signal. This algorithm is slow in processing but it has high reconstruction accuracy among the other algorithms. The remaining sections of this paper are organized as follows: section (2) gives the principle and featurs of CSD, section (3) introduces the mathematical analysis of CSD, section (4) explains the system model, numerical results and conclusion are explained in section (5) and (6) respectively.

\section{CYCLOSTATIONARY BASED DETECTION}

Cyclostationary based detection principle exploits statistical periodicity feature of the received signal where this feature is inherent feature of communication signals. The structure of this signal consists of carrier frequency synchronization words, OFDM cyclic prefixes, SS code, sampling rate, etc. Cyclic mean of the received signal or cyclic autocorrelation received signal is used to perform cyclostationary detection as first order or second order cyclostationary respectively [17]. Since the cyclic feature is not found in wide sense noise, hence it is used to detect the presence of primary user signal. This method is robust against noise uncertainty in addition to other good properties like no need to pre-information about primary user signal (incoherent), excellent recognition of several types of signals (BFSK, MFSK, QFSK, OFDM, CDMA) [10], but the main drawback of this technique is long process time and high complexity [18].

\section{MATHEMATICAL ANALYSIS OF CYCLOSTATIONARY}

The periodicity of the signal $\mathrm{x}(\mathrm{t})$ can be expressed as first order periodicity related to periodic mean of the signal $\mathrm{E}[\mathrm{x}(\mathrm{t})]$, or by both periodic mean and periodic autocorrelation of the signal $\mathrm{R}_{\mathrm{x}}(\mathrm{t}, \tau)$ as a second order periodicity. Both mean and autocorrelation of the signal are periodic function with specific time say $\mathrm{T}_{0}$, since the periodicity conditions are [17]:

$$
\begin{aligned}
& \mathrm{E}\left[\mathrm{x}\left(\mathrm{t}+\mathrm{T}_{0}\right)\right]=\mathrm{E}[\mathrm{x}(\mathrm{t})] \\
& R_{x}\left(\mathrm{~T}+\mathrm{t}_{0}, \tau\right)=R_{x}(\mathrm{t}, \tau)
\end{aligned}
$$

where

$$
R_{x}=\mathrm{E}[\mathrm{x}(\mathrm{t}+\tau) \mathrm{x}(\mathrm{t})]
$$

Using Fourier series coefficients, this shows that the cyclic autocorrelation function (CAF) can be expressed as the cyclic features of received signal [18]:

$$
R_{x x}^{*}(\mathrm{t}+\mathrm{T} / 2, \mathrm{t}-\mathrm{T} / 2)=\sum_{\alpha \in \mathrm{A}} \mathrm{R}_{\mathrm{xx}}{ }^{*}(\tau) e^{-\mathrm{j} 2 \pi \alpha \mathrm{t}}
$$

where:

$$
R_{x x}^{*}(\mathrm{t}+\mathrm{T} / 2, \mathrm{t}-\mathrm{T} / 2)
$$

This is the conjugate autocorrelation function. Then:

$$
R_{x x}^{\alpha}=1 / \mathrm{T}_{0} \int_{-T / 2}^{T / 2} R_{x x} *(\mathrm{t}+\mathrm{T} / 2, \mathrm{t}-\mathrm{T} / 2) e^{-\mathrm{j} 2 \pi \alpha \mathrm{t}} \mathrm{dt}
$$

$R_{x x}^{\alpha}$ : Cyclic autocorrelation function (CAF), $\alpha$ : cyclic frequency. 


\section{SYSTEM MODEL}

Hypothesis test problem can be used as a rule to detect the received signal $x(t)$ vacant band or occupied band by the primary user signal, which is the reconstructed signal by $\ell 1$ - norm algorithm as:

$$
\begin{aligned}
& \left.x(t)\right|_{H_{0}}: \text { stationary signal (PU signal is absent) } \\
& \left.x(t)\right|_{H_{1}}: \text { cyclostationary signal (PU signal is present) }
\end{aligned}
$$

That means the procedure is to search the absence of cyclic magnitude of autocorrelation function $(\mathrm{CAF})$ in the acquisition spectrum during a specific time period $\left(\mathrm{nT}_{0}\right)$, where $\mathrm{n}$ is a real number. The decision is taken then depending on comparison results of CAF with pre-calculated threshold $\lambda$. Windowing approach is used to perform cyclostationary system [18], which can be described as follows:

1) Calculate the cyclic frequency $(\alpha)$, window and FFT size.

2) Do the Fourier transform for each window segment of the signal.

3) Spectral correlation function $(\mathrm{CAF})$ is calculated for each frame.

4) Normalize each calculated CAF with mean.

5) Compare maximum of normalized CAF with a threshold.

6) 6-Absence or presence of primary user signal decision is taken depending on the result of step 5.

As below:

If $\max$ of $\mathrm{CAF} \geq \lambda$ the primary user signal is present

If max of $\mathrm{CAF}<\lambda$ the primary user signal is absent

The cyclostationary detector is shown in Figure 1 [18].

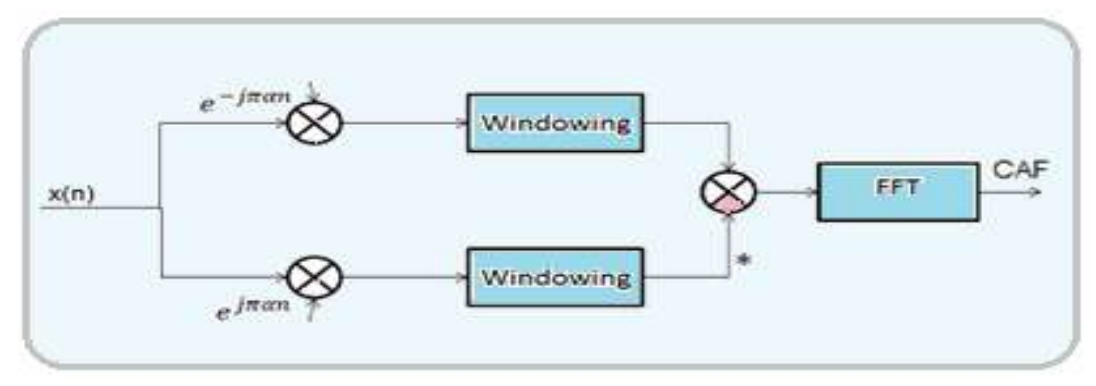

Figure 1. Cyclostationary detector

Probability of false alarm $\mathrm{P}_{\mathrm{fa}}$ happens when the primary signal is absent in the available spectrum but the system fancies that it is present and therefore the system allocates band to the SU. It happened when only noise power is larger than the threshold of sensing. This case is not desired and should be minimized [19]. Probability of detectin $P_{d}$ is detecting enough energy from the secondery user radio network when the primary user radio is actually request a spectrum sharing [20]. The probability of detection and the probability of false alarm can be expressed as [18].

$$
\begin{aligned}
& \mathrm{P}_{\mathrm{d}}=\mathrm{Q}\left[\sqrt{\frac{2 \gamma}{\sigma^{2}}}, \frac{\lambda}{\sigma_{B}}\right] \\
& \mathrm{P}_{\mathrm{fa}}=e^{-\left(\frac{(2 \mathrm{~N}+1) \lambda^{4}}{2 \sigma^{4}}\right)}
\end{aligned}
$$

where:

$$
\begin{aligned}
& \lambda=\sqrt{-\left(\left[2 \sigma^{4} /(2 \mathrm{~N}+1)\right] * \log \left(P_{f a}\right)\right.} \\
& \sigma_{\mathrm{B}}=\frac{(2 \gamma+1) \sigma^{\wedge} 4}{(2 \mathrm{~N}+1)}
\end{aligned}
$$

$\sigma^{2}$ : Variance of received signal, $\lambda$ : Threshold $\mathrm{N}$ : Number of sample, $\gamma$ : signal to noise ratio SNR 
To reduce the acquisition time of the received signal spectrum compressive sensing technique takes small number elements vector of measurements (y) with $(M)$ elements from the wideband signal that has large elements $(\mathrm{N})$, where $\mathrm{N} \gg \mathrm{M}$. Measurements vector has sampling rate less than Nyquist rate sampling (sub-Nyquist).This vector is expressed as:[21]

$$
y=\phi x
$$

where: $y$ is measurement vector, $\phi$ : is sensing matrix, $x$ : Nyquist rate secondary user signal.

Sensing matrix must be designed under important conditions. The most important of them is; restricted isometry property (RIP) and incoherency between sensing matrix and spares representation basis matrix. These conditions are the key parameters to reconstruct the original signal from the measurements vector $y[22]$.

Recovery algorithm used to reconstruct the original signal is based on linear programing processing where the system is considered as underdetermined system [22]. This problem is converted into convex optimization and can be solved as linear programing problem:

$$
\hat{x}=\operatorname{argmin}\|x\| \quad \text { Subjected to } y=\phi x
$$

Figure 2 shows the block diagram of the suggested system.

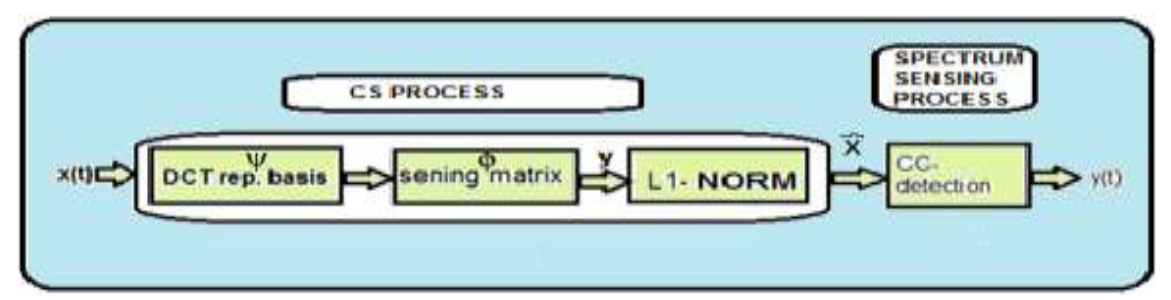

Figure 2. Cyciostationary based detection with compressive sensing

\section{NUMERICAL RESULTS AND DISCUSSION}

Monte Carlo computer simulation is used to simulate the procedure of the system to calculate the probability of detection $\mathrm{P}_{\mathrm{d}}$ over AWGN channel with constant $\mathrm{P}_{\mathrm{fa}}=0.01$. BFSK signal and low range of SNR is used. Cyclostationary compressive sensing detector is performed by reconstructing the original signal from y vector, Then dividing this signal to overlapped frames with overlap value $=3 / 4$. Hamming window is used with length 256 samples to calculate the CAF by computer simulation with 6000 sample divided to 100 frames. The probability of detection is calculated as a function of SNR for several compression ratios $\mathrm{M} / \mathrm{N}$. Conventional cyclostationary based detection can be obtained by making compression ratio of compressive sensing $(\mathrm{M} / \mathrm{N}=1)$ to give a results that are the same as no compressive sensing. Figure 3 shows the coventional cyclostationary detection performance by showing the probability of detection as a function of SNR. Both simulation and theoretical results are shown in order to compare with cyclostationary detector with compressive sensing. Using compressive sensing technique with cyclostationary detector with several compression ratios $(\mathrm{M} / \mathrm{N})$ to investigate the effect of compressive sensing process on cyclostationary performance.

Figure 4 shows the detection performance of cyclostationary with compressive sensing with $(\mathrm{M} / \mathrm{N}=0.15)$. It is clear that even if the compressive sensing with very low compression ratio (about $\mathrm{M} / \mathrm{N}=0.15$ ) the performance of the system is still accepted with very small degradation and close to the conventional performance case at the same range of SNR and $\mathrm{P}_{\mathrm{fa}}$.

Figure 5 shows the effect compression ratio $\mathrm{M} / \mathrm{N}$ as function of the probability of detection and SNR for several values of $\mathrm{M} / \mathrm{N}$. The effect of $\mathrm{M} / \mathrm{N}$ starts to affect the performance with range below $\mathrm{M} / \mathrm{N}=0.15$. This effect is decreased as $\mathrm{M} / \mathrm{N}$ increases and still with minimum effect when $\mathrm{M} / \mathrm{N}$ is close to (0.2). 


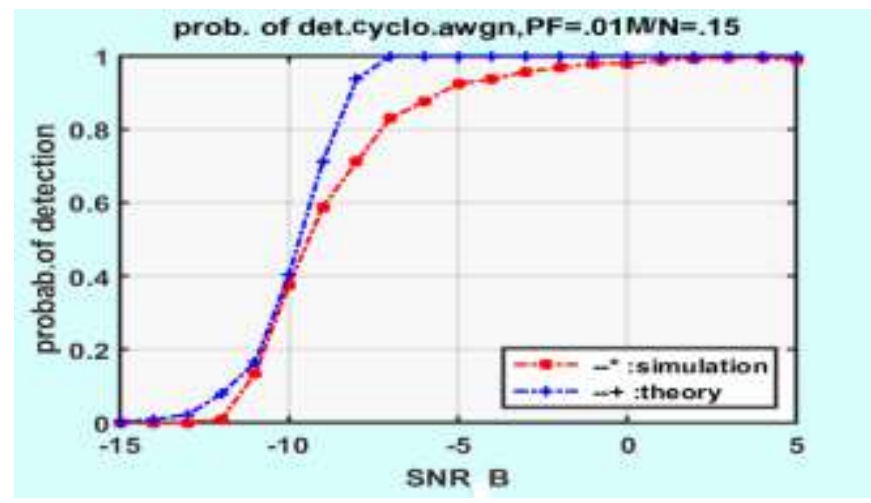

Figure 3. Coventional cyclostationary detection over AWGN channel

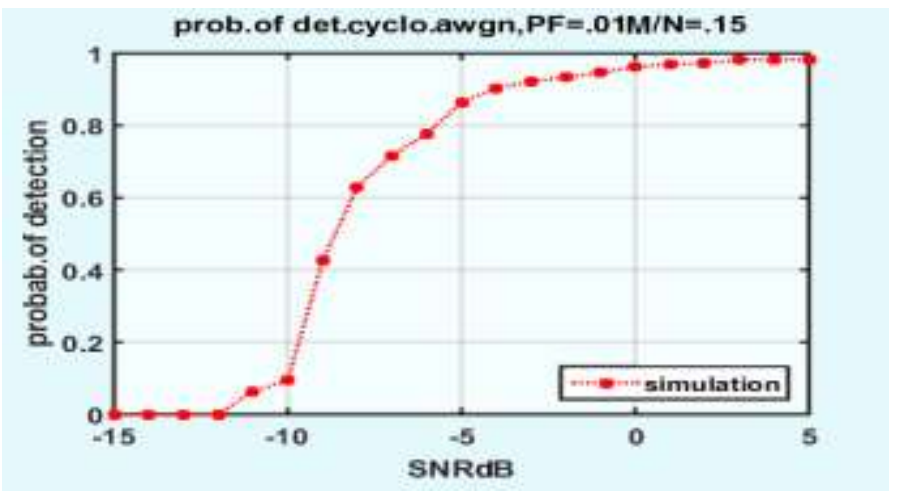

Figure 4. Cyclostationary detection with compressive sensing $(\mathrm{M} / \mathrm{N}=0.15)$ over AWGN channel

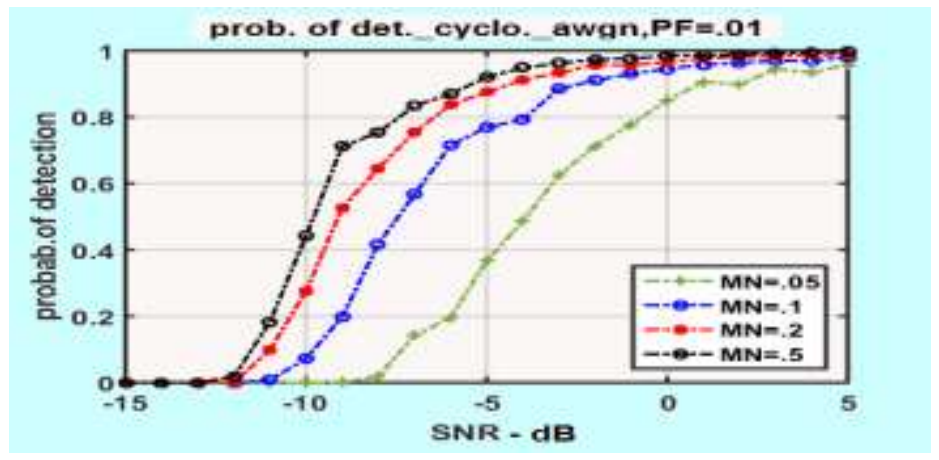

Figure 5. Probability of detection versus SNR for several M/N

This indicates that even if the reconstruction error of the recovery algorithm of compressive sensing has large values when the reconstructed signal has relatively large distortion, the cyclostationary features of the signal is still distinguishable, and CAF can be calculated with high efficiency by the proposed detector. Table 1 shows that to get $P_{d}$ close to 0.9 the required SNR is increased from $(-5.4 \mathrm{~dB})$ to $(0.91 \mathrm{~dB})$ when the compression ration $\mathrm{M} / \mathrm{N}$ is decreased from $(0.5)$ to $(0.05)$. Also Table 2 shows the obtained probability of detection $\mathrm{P}_{\mathrm{d}}$ versus $\mathrm{M} / \mathrm{N}$ for a constant $(\mathrm{SNR}=-4 \mathrm{~dB})$. Again in this table $\mathrm{P}_{\mathrm{d}}$ is increased with increasing $\mathrm{M} / \mathrm{N}$.

Table 1. M/N VS SNR for $\mathrm{P}_{\mathrm{d}}=0.9$

\begin{tabular}{ccccc}
\hline $\mathrm{M} / \mathrm{N}$ & 0.05 & 0.1 & 0.2 & 0.5 \\
\hline $\mathrm{SNR}(\mathrm{dB})$ & 0.91 & -2.49 & -4.3 & -5.4 \\
\hline
\end{tabular}


Table 2. $\mathrm{M} / \mathrm{N}$ VS $\mathrm{P}_{\mathrm{d}}$ for $\mathrm{SNR}=-4 \mathrm{~dB}$

\begin{tabular}{ccccc}
\hline $\mathrm{M} / \mathrm{N}$ & 0.05 & 0.1 & 0.2 & 0.5 \\
\hline $\mathrm{P}_{\mathrm{d}}$ & 0.48 & 0.79 & 0.91 & 0.94 \\
\hline
\end{tabular}

Figure 6 shows the very small effect of compression process to $\mathrm{P}_{\mathrm{fa}}$ of CSD technique, where every level of compression between $\mathrm{M} / \mathrm{N}=1$ to $\mathrm{M} / \mathrm{N}=0.25$ with $\mathrm{P}_{\mathrm{d}}=0.9$ needs to be increased at SNR level from $-7.75 \mathrm{~dB}$ to $-5.75 \mathrm{~dB}$ to decrease $\mathrm{P}_{\mathrm{fa}}$ from 1 to 0 .

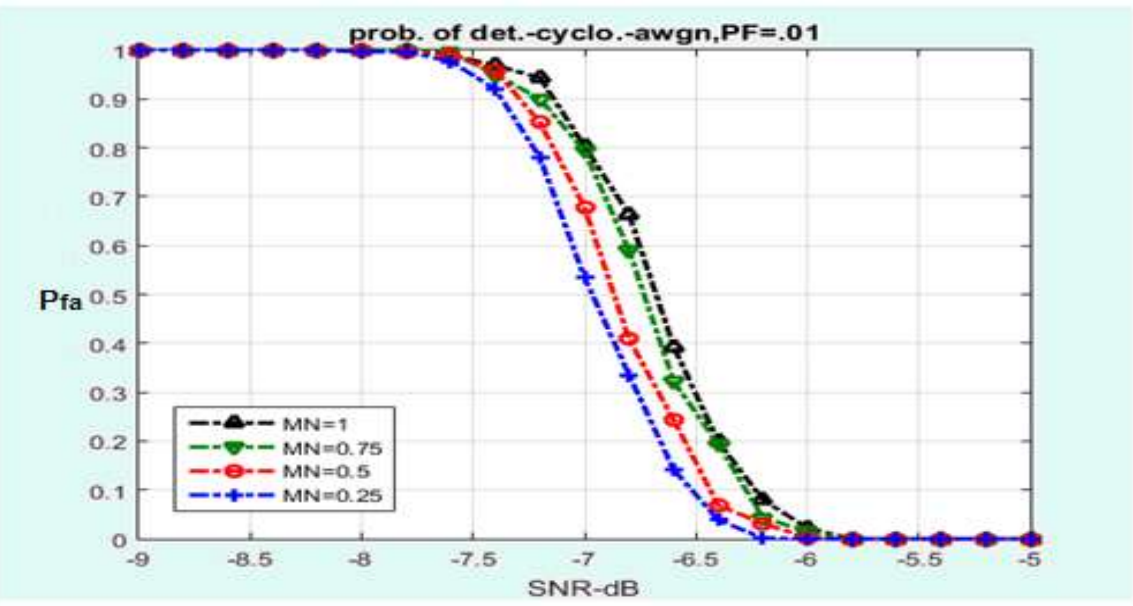

Figure 6. Probability of false alarm versus SNR for several M/N

Reconstruction error of the recovery algorithm can be explained by several ways like mean square error (MSE), reconstruction error $\mathrm{R}_{\mathrm{e}}$ or by checking the similarity between the original signal with the reconstructed signal using correlation coefficient factor $\mathrm{C}_{\mathrm{cs}}$ [18]. Reconstruction error is the ratio between the norm of difference between the two signals (recovered signal \& original signal) to the norm of the original signal as [23]:

$$
\mathrm{R}_{\mathrm{e}}=\frac{\|\hat{x}-x\|}{\|x\|}
$$

Figure 7 shows the reconstruction error effect versus $\mathrm{M} / \mathrm{N}$.

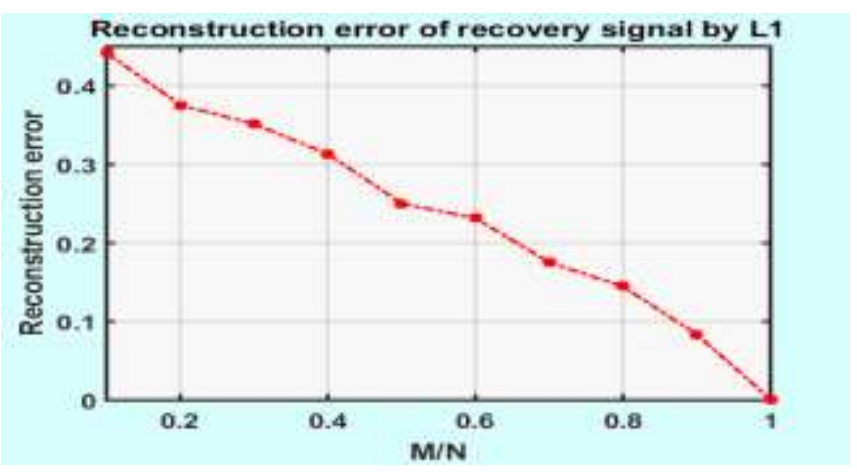

Figure 7. Reconstruction error of recovery signal by $\ell 1$-norm algorithm

Mean square error (MSE) is expressed as: [24]

$$
\operatorname{MSE}=\frac{1}{N} \sum_{N}(x-\hat{x})^{2}
$$


Figure 8 shows the MSE effect versus M/N.

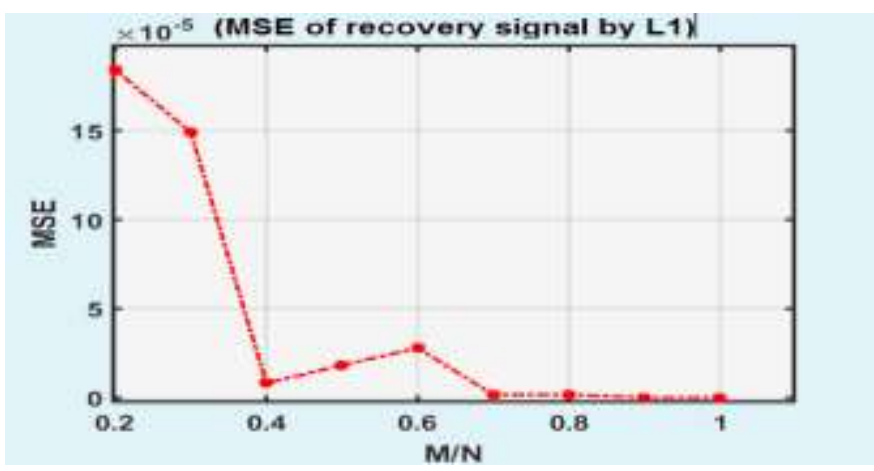

Figure 8. MSE versus compression ratio for recovery signal by $\ell 1$-norm algorithm

The processing time is related directly to the $\mathrm{M} / \mathrm{N}$, since $\mathrm{M} / \mathrm{N}$ with very small values meet good detection performance so that the processing time is decreased with the same range of $\mathrm{M} / \mathrm{N}$ to very small value related to the sensing time required to operate with the whole wideband signal. This is shown clearly in Figure 9.

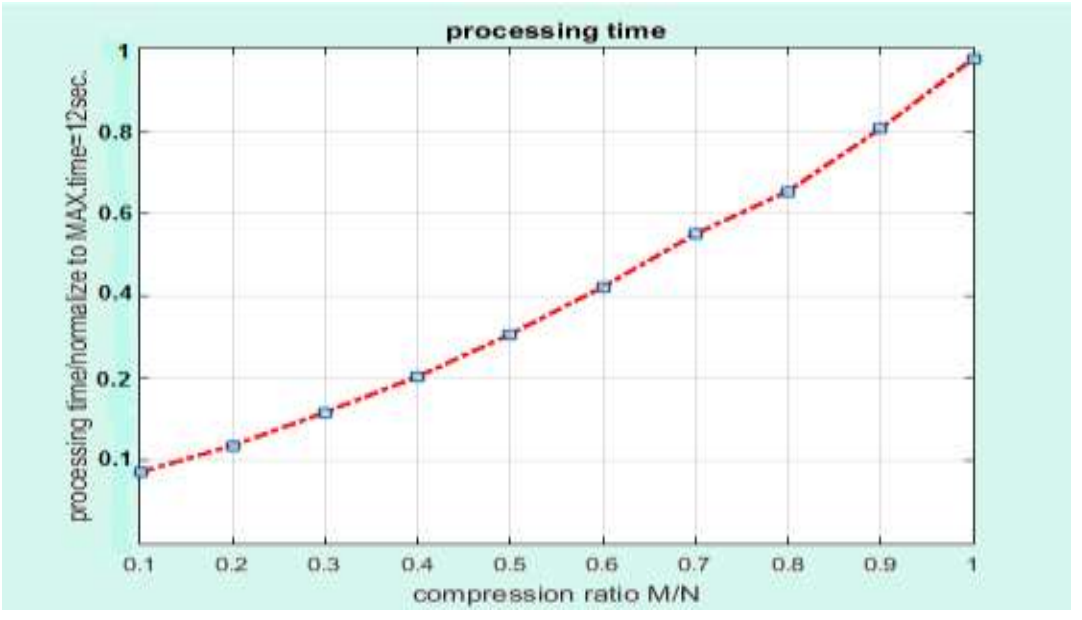

Figure 9. Processing time versus compression ratio $\mathrm{M} / \mathrm{N}$

\section{CONCLUSION}

This paper studied the spectrum sensing techniques with wideband signal. Cyclostationary detection with compressive sensing technique is discussed where the received signal is sampled at very low rate (0.15 of Nyquist rate) by using DCT basis and random Gaussian sensing matrix to obtain the measurement vector which has low sampling rate. From the simulation results, it is clear that cyclostationary detector has excellent performance when combined with compressive sensing to deal with wideband signal even if the compression ratio is very small. This is very important issue which leads to reduce the processing time largely to achieve quick signal acquisition then achieving, accurate and efficient usage of available spectrum. The sensing time is very important parameter that acts approximately at all aspects of spectrum sensing process and cognitive radio system functions.

\section{ACKNOWLEDGEMENTS}

The authors wish to thank the staff of electrical engineering dept, college of engineering, Al-Mustansiriyah University for supporting this work. 


\section{REFERENCES}

[1] A. Gorcin, et al., "AnAdaptive Threshold Method for Spectrum Sensing in Multi-Channel Cognitive Radio Networks," 17th International Conference on Telecommunications, 2010.

[2] M. S. Hossain, et al., "Energy detection performance of spectrum sensing in cognitive radio," IJ Information Technology and Computer Science, vol. 11, pp. 11-17, 2012.

[3] D. Pineda and C. Hernandez, "Cognitive radio for TVWS usage," TELKOMNIKA Telecommunication Computing Electronics and Control, vol. 17, no. 6, 2019.

[4] Y. Zhou, et al., "FSK-Signal Detection in Cognitive Radios Using First-Order Cyclostationary," 17th International Conference on Telecommunications, 2010.

[5] E. Hossain, et al., "Dynamic Spectrum Access and Management in Cognitive Radio Networks," United States of America by Cambridge University Press, New York, 2009.

[6] S. Atapattu, et al., "Energy Detection for Spectrum Sensing in Cognitive Radio," Springer New York, Heidelberg Dordrecht London, 2014.

[7] F. F. Digham, et al., "On the energy detection of unknown signals over fading channels," in IEEE International Conference on Communications, 2003.

[8] M. Ranjeeth and S. Anuradha, "Performance of fading channels on energy detection based spectrum sensing," Procedia Materials Science, vol. 10, pp. 361-370, 2015.

[9] W. A. Gardner, "Exploitation of spectral redundancy in cyclostationary signals," in IEEE Sig. Proc. Mag., vol. 8, pp. 14-36, 1991.

[10] P. S. Aparna and M. Jayasheela, "Cyclostationary feature detection in cognitive radio using different modulation schemes," International Journal of Computer Applications, vol. 47, no. 21, 2012.

[11] S. M. Kay, "Fundamentals of Statistical Signal Processing: Estimation Theory," Englewood Cliffs, NJ, PrenticeHall, 1993.

[12] S. Dannana, et al., "Spectrum Sensing Using Matched Filter Detection," Springer Nature Singapore Pte Ltd., 2018.

[13] E. Candues, et al., "Robust uncertainty principles: Exact signal reconstruction from highly incomplete frequency information," IEEE Trans. Inform. Theory, vol. 52, no. 2 pp. 489-509, 2006.

[14] D. Donoho, "Compressed sensing," IEEE Trans. Inform. Theory, vol. 52, no. 4, 2006.

[15] M. A. Davenport, et al., "Detection and Estimation with Compressive Measurements," Rice university, Technical Report TREE 0610 November 1, 2006.

[16] F. Salahdine, "Compressive Spectrum Sensing for Cognitive Radio Networks," PhD degree of National Institute of Posts and Telecommunications, 2018.

[17] S. G. Mohapatra, et al., "Cyclostationary based windowing method for spectrum sensing in cognitive radio network," 2013 International Conference on Information Communication and Embedded Systems (ICICES), 2013.

[18] M. B. Dave, "Spectrum Sensing in Cognitive Radio: Use of Cyclo-Stationary Detector," Dept. Electronics and Comm. Eng., National Institute of Technology, 2012.

[19] A. S. Khobragade and R. D. Raut, "Hybrid Spectrum Sensing Method for Cognitive Radio," International Journal of Electrical and Computer Engineering, vol. 7, no. 5, p. 2683, 2017.

[20] M. A. Saad, et al., "Spectrum sensing and energy detection in cognitive networks," Indonesian Journal of Electrical Engineering and Computer Science, vol. 17, no. 1, pp. 465-472, 2020.

[21] J. Haupt and R. Nowak, "Compressive sampling for signal detection," Proceeding of the IEEE International conf. on Acoustics, Speech and Signal Proc. (ICASSP '07), vol. 3, pp. 1509-1512, 2007.

[22] I. Alkhouri, "Spectrum Sensing Based OnCompressdSampling," San Diego University, 2013.

[23] Y. Arjoune, et al., "Compressive sensing: Performance comparison of sparse recovery algorithms," 2017 IEEE 7th annual computing and communication workshop and conference (CCWC), 2017.

[24] E. C. Marques, et al., “A review of sparse recovery algorithms,” IEEE Access, vol. 7, pp. 1300-1322, 2018.

\section{BIOGRAPHIES OF AUTHORS}

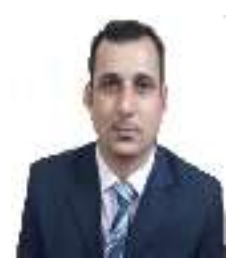

Ali Mohammad A.AL-Hussain senior engineer working in ministry of electricity (MOE-IRAQ), currently he is Msc student in Elec. Eng. Dept., college of Engineering, Al Mustansiriyah university, Baghdad, Iraq.

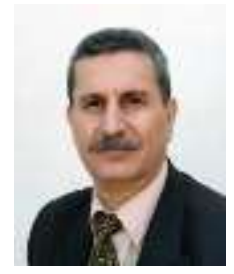

Professor Maher K. Mahmood Al-Azawi works as a head of communication group in Elec. Eng. Dept., college of Engineering, Al Mustansiriyah university, Baghdad, Iraq. His publications exceeds 50 papers in local and international journal and conferences. 